\title{
Fiber-Centered Granger Causality Analysis
}

\author{
Xiang $\mathrm{Li}^{1}$, Kaiming $\mathrm{Li}^{1,2}$, Lei Guo ${ }^{2}$, Chulwoo $\mathrm{Lim}^{1}$, and Tianming $\mathrm{Liu}^{1}$ \\ ${ }^{1}$ Department of Computer Science and Bioimaging Research Center, \\ The University of Georgia, Athens, GA \\ ${ }^{2}$ School of Automation, Northwestern Polytechnic University, Xi' an, China
}

\begin{abstract}
Granger causality analysis (GCA) has been well-established in the brain imaging field. However, the structural underpinnings and functional dynamics of Granger causality remain unclear. In this paper, we present fibercentered GCA studies on resting state fMRI and natural stimulus fMRI datasets in order to elucidate the structural substrates and functional dynamics of GCA. Specifically, we extract the fMRI BOLD signals from the two ends of a white matter fiber derived from diffusion tensor imaging (DTI) data, and examine their Granger causalities. Our experimental results showed that Granger causalities on white matter fibers are significantly stronger than the causalities between brain regions that are not fiber-connected, demonstrating the structural underpinning of functional causality seen in resting state fMRI data. Cross-session and cross-subject comparisons showed that our observations are reproducible both within and across subjects. Also, the fiber-centered GCA approach was applied on natural stimulus fMRI data and our results suggest that Granger causalities on DTI-derived fibers reveal significant temporal changes, offering novel insights into the functional dynamics of the brain.
\end{abstract}

Keywords: Granger Causality Analysis, fMRI, DTI, Time Series, Brain State.

\section{Introduction}

Proposed by Clive Granger in 1969 (Granger 1969), Granger causality analysis (GCA) has been widely applied to analyze the relationships between time series. Briefly, a time series $\mathrm{X}$ is said to Granger-cause time series $\mathrm{Y}$ if the values of $\mathrm{X}$ provide statistically significant information about future values of Y. The GCA is very useful in functional MRI (fMRI) signal analysis, since different brain regions are supposed to connect together and have causal influence upon each other. Thus in recent years, it has been widely used in the brain imaging field [2-4], in order to obtain a hierarchical understanding of the interaction and correlation between different brain regions.

Despite wide application of GCA in fMRI, however, the structural underpinnings of GCA remain unclear, e.g., how structural connectivity is related to Granger causality? In addition, existing approaches of GCA on brain networks [2-4] assume temporal stationarity, where Granger causalities are computed over the entire scan and used to characterize the causality across regions. However, accumulating literature [e.g., 10], have shown that functional brain connectivity is under dynamical changes. Other literature has reported decoding brain states using sliding-window approach [12]. In responses to the above-mentioned issues, this paper presents a fiber-centered GCA 
approach to examine resting state and natural stimulus fMRI datasets, in order to elucidate the structural substrates and functional dynamics. Specifically, we extract the fMRI BOLD signals from the two ends of a DTI-derived fiber, and measure their Granger causalities. Our premise is that as axonal fibers are the structural substrates of functional connections between computational centers of cortical regions, the fMRI time series along the fibers should reflect the functional causality between brain regions, if any such functional causality exists.

Our experimental results showed that Granger causalities on white matter fibers are significantly stronger than the causalities between brain regions that are not connected by fibers, suggesting the structural underpinning of functional causality observed in resting state fMRI data. This observation is replicated in different imaging sessions of the same subject and across individual subjects. In addition, our experimental results of applying the fiber-centered GCA approach on natural stimulus fMRI data suggest that Granger causalities on fibers reveal significant temporal changes, offering novel insights into the functional dynamics of the brain.

\section{Method}

\subsection{Overview of the Method}

Fig. 1 shows the flowchart of this analysis pipeline. The GCA model is akin to the work flow of the program "MATLAB toolbox for Granger causal connectivity"

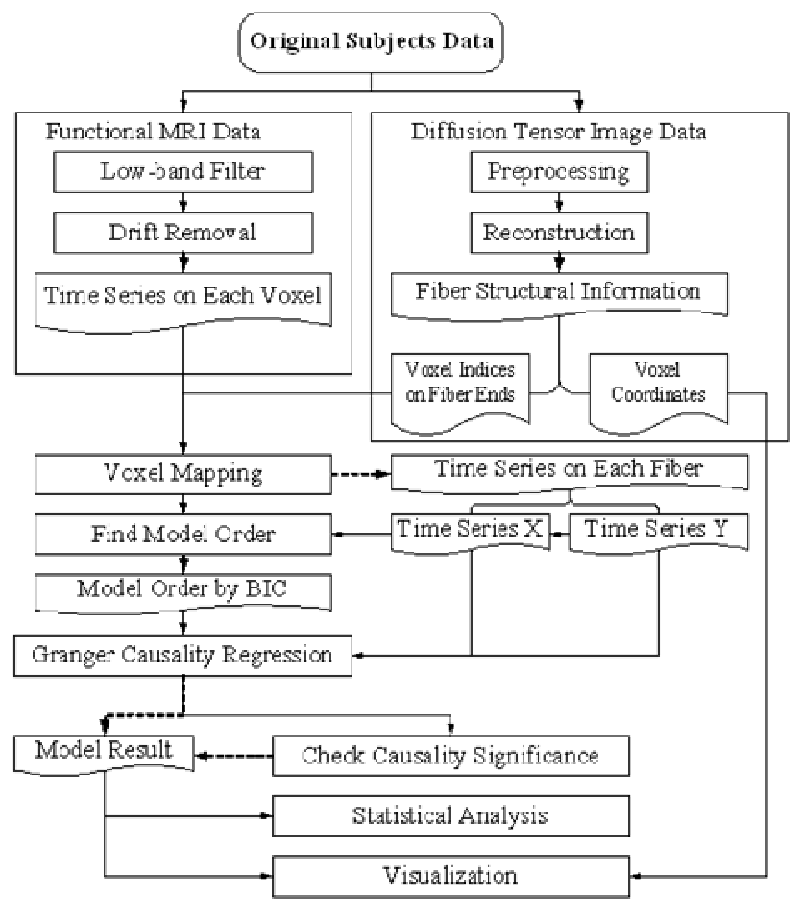

Fig. 1. The flowchart of the fiber-centered GCA framework 
developed by Anil Seth (Seth 2005) and in previous studies in applying GCA to EEG data (Andreas, Dean et al. 2009). Details of other methodology components are described in Section 2.2 and 2.3.

\subsection{Data Acquisition and Preprocessing}

A 3T GE Signa MRI system was used for data acquisition of fMRI data analyzed in this study: resting-state and natural-stimulus fMRI data. Resting state fMRI data were acquired with dimensionality $128 * 128$ (matrix)*60 (slices)*100 (volumes), in-plane resolution $2 \mathrm{~mm} * 2 \mathrm{~mm} * 2 \mathrm{~mm}$ isotropic, TR $5 \mathrm{~s}$, TE $25 \mathrm{~ms}$, and flip angle 90 degrees. DTI data were acquired at the same spatial resolution, with a $15.5 \mathrm{~s}$ TR, TE of $89.5 \mathrm{~ms}$, and generated 30 gradient channel DWI volumes. In the natural stimulus fMRI scan, we randomly selected video shots from the TRECVID 2005 database [11], which were presented to the subject during a two-session scan. The acquisition parameters were as follows: dimensionality $128 * 128 * 60 * 240$, spatial resolution $2 \mathrm{~mm} * 2 \mathrm{~mm} * 2 \mathrm{~mm}$, TR $5 \mathrm{~s}$, TE 25ms, and flip angle 90.

For preprocessing, we registered fMRI data to the DTI space by the FSL FLIRT tool. It should be noted that because DTI and fMRI sequences are both echo planar imaging (EPI) sequences, their distortions tend to be similar [7]. So the misalignment between DTI and fMRI images is much less than that between T1 and fMRI images [7]. DTI pre-processing included skull removal, motion correction and eddy current correction. Then fiber tracking was performed using MEDINRIA. Brain tissue segmentation was conducted on DTI data by a similar method in [8] and the cortical surface was reconstructed using the marching cubes algorithm. FMRI preprocessing steps included motion correction, spatial smoothing, temporal prewhitening, slice time correction, global drift removal, and band pass filtering. After the above preprocessing, we used white matter fibers to guide the fiber-centered GCA, which has been applied in previous studies on brain network and convincing results were obtained, showing the feasibility of the inter-modality data registration [13]. In our work, fMRI BOLD signals of the grey matter voxel pairs connected by a white matter fiber were extracted for the Granger causality analysis, as illustrated in Fig. 2. The number of voxel pairs connected by each fiber varies in different datasets. In average case there are tens of voxel pairs connected by one fiber.
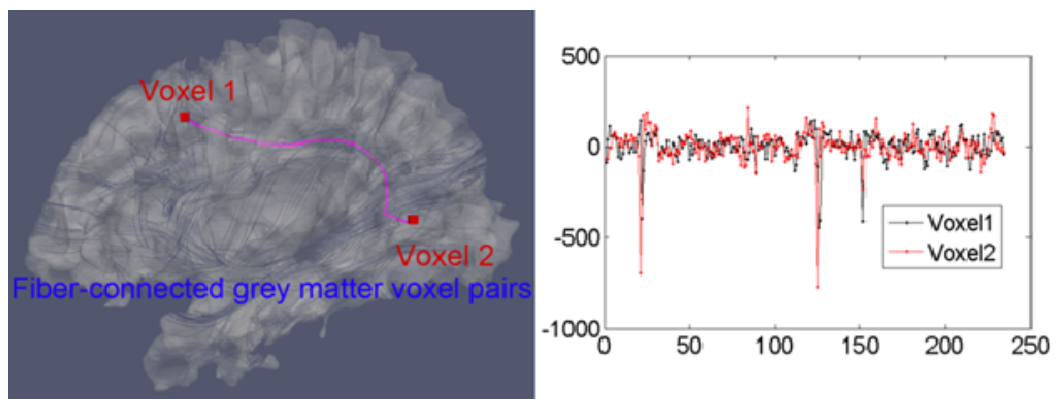

Fig. 2. Two grey matter voxels (marked in red) connected by a fiber (in purple). fMRI time series at these two voxels are shown in the right figure. 


\subsection{Granger Causality Analysis}

Given two stochastic processes $\mathrm{X}$ and $\mathrm{Y}$, if they are stationary, each of the process can be expressed as an auto-regression of their lagged values:

$$
\begin{aligned}
& \mathrm{X}_{\mathrm{t}}=\sum_{\mathrm{i}=1}^{\mathrm{P}} \mathrm{a}_{\mathrm{i}} \mathrm{X}_{\mathrm{t}-\mathrm{i}}+e 1_{\mathrm{t}} \\
& \mathrm{Y}_{\mathrm{t}}=\sum_{\mathrm{i}=1}^{\mathrm{P}} \mathrm{d}_{\mathrm{i}} \mathrm{Y}_{\mathrm{t}-\mathrm{i}}+e 2_{\mathrm{t}}
\end{aligned}
$$

where e 1 and e 2 are prediction errors and their variances describe the accuracy of the prediction. Assume they have potential causality influences upon each other, there is:

$$
\begin{aligned}
& \mathrm{X}_{\mathrm{t}}=\sum_{\mathrm{i}=1}^{\mathrm{P}} \mathrm{a}_{\mathrm{i}} \mathrm{X}_{\mathrm{t}-\mathrm{i}}+\sum_{\mathrm{i}=1}^{\mathrm{P}} \mathrm{b}_{\mathrm{i}} \mathrm{Y}_{\mathrm{t}-\mathrm{i}}+e 3_{\mathrm{t}} \\
& \mathrm{Y}_{\mathrm{t}}=\sum_{\mathrm{i}=1}^{\mathrm{P}} \mathrm{c}_{\mathrm{i}} \mathrm{X}_{\mathrm{t}-\mathrm{i}}+\sum_{\mathrm{i}=1}^{\mathrm{P}} \mathrm{d}_{\mathrm{i}} \mathrm{Y}_{\mathrm{t}-\mathrm{i}}+e 4_{\mathrm{t}}
\end{aligned}
$$

where e 3 and e 4 are prediction errors and $\mathrm{a}, \mathrm{b}, \mathrm{c}, \mathrm{d}$ are linear regression coefficients. In order to study the dependency between $\mathrm{X}$ and $\mathrm{Y}$, the null hypothesis $\mathrm{H} 0$ : $\{b\}=0$ was made, which means $\mathrm{Y}$ will not significantly cause $\mathrm{X}$. According to the null hypothesis, we can construct the F-statistics:

$$
\mathrm{F}_{\mathrm{Y} \rightarrow \mathrm{X}}=\frac{\operatorname{var}(e 1)-\operatorname{var}(e 3)}{\operatorname{var}(e 3)}
$$

When there is no causality caused by $\mathrm{Y}$ to $\mathrm{X}$, the value of $\mathrm{F}_{\mathrm{Y} \rightarrow \mathrm{X}}$ will approach zero since the additional $\mathrm{Y}$ terms will not influence the explanation power in Eq. (3). And if the value is greater than the given threshold, we will reject the null hypothesis, which means there is a significant causality caused by $\mathrm{Y}$ to $\mathrm{X}$.

The original GCA model only gives the result of whether there is causality, which is limited for the brain imaging research since there are reciprocal polysynaptic connections between brain areas (Friston 2009). Here we applied the conditional GCA (Seth 2005) which was able to evaluate the Causality Magnitude (CM):

$$
\mathrm{CM}_{\mathrm{Y} \rightarrow \mathrm{X}}=\ln \left(\frac{\operatorname{var}(e 3)}{\operatorname{var}(e 1)}\right)
$$

This value is used in the following analysis to evaluate the strength of Granger causality; higher CM value indicates greater causal influence.

In this study, we applied the above results obtained by performing GCA on time series extracted from voxel pairs to study the causal characteristic of fibers that connects them. Since more than one voxel pairs may be connected by each fiber, the causality magnitude of that fiber is defined by: 


$$
\mathrm{CM}_{\text {fiber i }}=\left(\sum_{\mathrm{v} 1, \mathrm{v} 2 \in \mathrm{V}_{\mathrm{i}}} \mathrm{CM}_{\mathrm{v} 1 \rightarrow \mathrm{v} 2}+\mathrm{CM}_{\mathrm{v} 2 \rightarrow \mathrm{v} 1}\right) / \operatorname{sizeof}\left(\mathrm{V}_{\mathrm{i}}\right)
$$

where $\mathrm{V}_{\mathrm{i}}$ is the set of voxel pairs connected by the ith fiber, and $\mathrm{v} 1$, v2 are pair of voxels in that set. The causality magnitude of fibers is in the range of $(0,2)$.

The F-statistics was used to determine whether there is a significant (given $\mathrm{P}=0.01$ ) causal connectivity between pair of voxels, thus a fiber connecting any one pair of voxels with significant causal connectivity at any direction is considered to be significantly casual-connected. Also, we defined the causality phase (CP) based on the F-statistics and the direction of the connectivity:

$$
\mathrm{CP}_{\text {voxel v1,v2 }}=\left\{\begin{array}{c}
1, \text { if causality from } \mathrm{v} 1 \text { to } \mathrm{v} 2 \text { is significant } \\
-1, \text { if causality from } \mathrm{v} 2 \text { to } \mathrm{v} 1 \text { is significant } \\
0, \text { if there is no significant causality }
\end{array}\right.
$$

And

$$
\mathrm{CP}_{\text {fiber i }}=\left(\sum_{\mathrm{v} 1, \mathrm{v} 2 \in \mathrm{V}_{\mathrm{i}}} \mathrm{CP}_{\mathrm{v} 1, \mathrm{v} 2}\right) / \operatorname{sizeof}\left(\mathrm{V}_{\mathrm{i}}\right)
$$

where $\mathrm{V}_{\mathrm{i}}$ is the set of voxel pairs connected by the ith fiber, and $\mathrm{v} 1$, v2 are a pair of voxels in that set. The causality phase of a fiber is in the range of $(-1,1)$.

\section{Results}

\subsection{Results in Resting State fMRI Data}

Totally, resting state fMRI dataset of 12 imaging sessions from 9 subjects was analyzed. We selected significantly casual-connected fibers and visualized the causality magnitude of them in Fig. 3. In this study, subject \#1, \#2 and \#3 had two scanning sessions, both under the same experimental circumstances. The data collected from two sessions of the same subject enabled us to compare the results and to see whether the inferred causalities were stable within subjects. As shown in Fig.3, the structures of significantly casualconnected fibers were almost identical between two sessions of all the three subjects, suggesting that certain fibers that were significantly casual-connected, might had more importance in causality connection than other fibers. Also, it could be seen that casualconnected fibers formed certain pattern in the brain, especially around frontal lobe and visual cortex in all the subjects, which indicated stronger connection and causal related activities (such as control and motivation) in these regions. Finally, as the statistics analysis of the results listed in Table 1 showed, the number of significantly causalconnected, as well as the causality magnitude, varied very little between two sessions. This provided good evidence that fiber-centered GCA on a whole-brain scale was highly reproducible within-subject.

Table 1. Similarity analyses between two sessions of each subject;

\begin{tabular}{c|c|c|c|c|c} 
& $\begin{array}{c}\text { Fibers } \\
\text { Tota1 }\end{array}$ & $\begin{array}{c}\text { Causa1-connected } \\
\text { fibers in session 1 }\end{array}$ & $\begin{array}{c}\text { Causa1-connected } \\
\text { fibers in session 2 }\end{array}$ & $\begin{array}{c}\text { Mean of CM in } \\
\text { session 1 }\end{array}$ & $\begin{array}{c}\text { Mean of CM in } \\
\text { session 2 }\end{array}$ \\
\hline Subject1 & 13073 & 622 & 589 & 0.48 & 0.48 \\
\hline Subject2 & 17671 & 1054 & 1053 & 0.49 & 0.49 \\
\hline Subject3 & 13482 & 1067 & 966 & 0.49 & 0.5
\end{tabular}




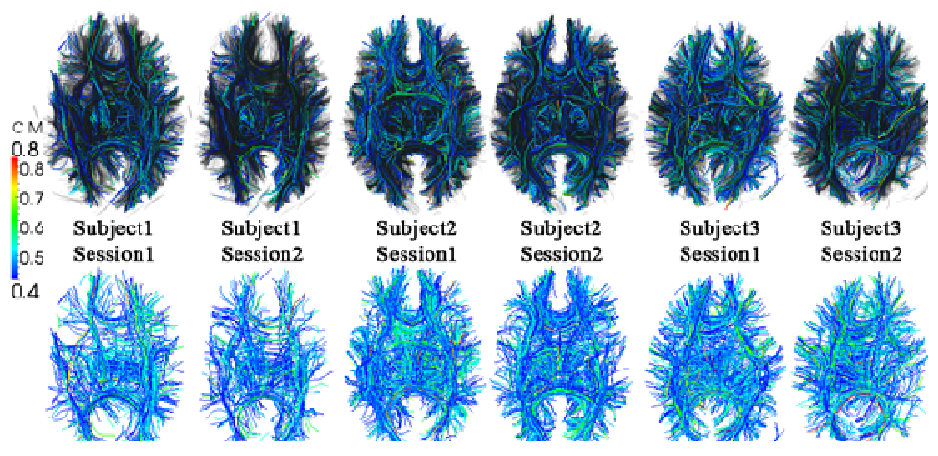

Fig. 3. Visualization of significant casual-connected fibers of three subjects (2 sessions). Images at the first and second row are identical, except that fibers that are not significantly causal-connected were colored in black in first row, and were removed in second row.

\subsection{Reproducibility Study: Cross-subjects}

In addition to the within-subject reproducibility (stability) study of our method, a cross-subjects reproducibility study was also conducted, to see whether the results were consistent. Fig. 4 showed the significantly casual-connected fibers of subject \#4 to \#9. It can be seen in the figure that there are consistency of causality pattern across subjects, despite the fact that fiber shapes and global causality levels varied among different subjects. For example, subject 9 had higher causality magnitude than subject 5 (the average CM of subject 9 is 0.54 , while subject 5 is 0.45 ), but the structures of the significantly casual-connected fibers were similar, suggesting the relatively stable and consistent oscillation architecture of the human brain in resting state.

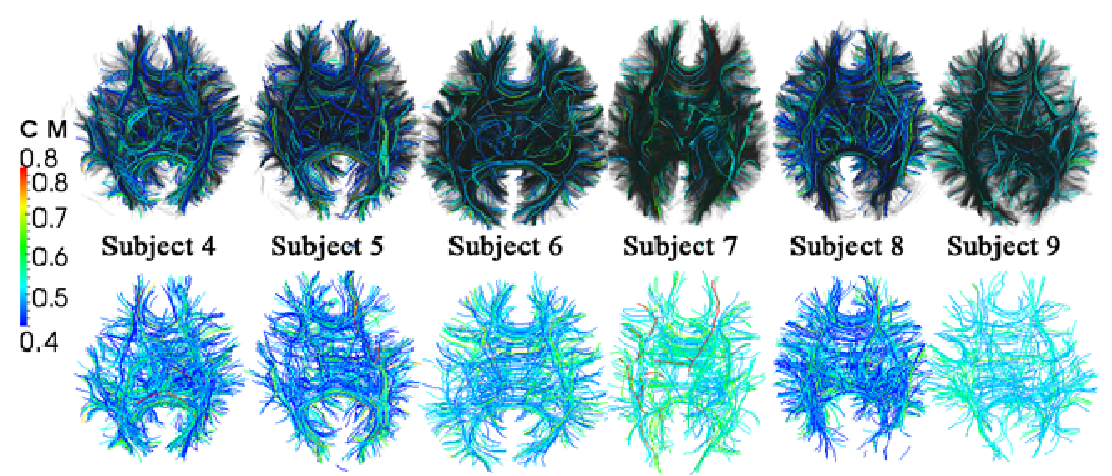

Fig. 4. Visualization of significant casual-connected fibers in 6 subjects. Images at the first and second row are identical, except that fibers that are not significantly causal-connected were colored in black in first row, and were removed in second row for comparison. 


\subsection{Granger Causality Dynamics in Natural Stimulus fMRI Data}

In this study, we also applied sliding window approach on time series scanned from natural stimulus fMRI data, and studied temporal change of causality strength and phase along sliding windows. We constructed 223 consecutive sliding windows with the length of 13 time points and perform GCA on voxel pairs connected by fibers, obtained causality magnitude as well as causality phases. The temporal dynamics of causality magnitude on all the fibers of a subject are shown in Fig. 5. We can see that the causality magnitude varies through the time, and forms patterns by the majority of fibers. Statistically, between each sliding window, the causality magnitude of a fiber will change $14.76 \%$ on average, and can be as high as $172 \%$, indicating dramatic temporal dynamical change. This result is reproducible in other 4 subjects analyzed.

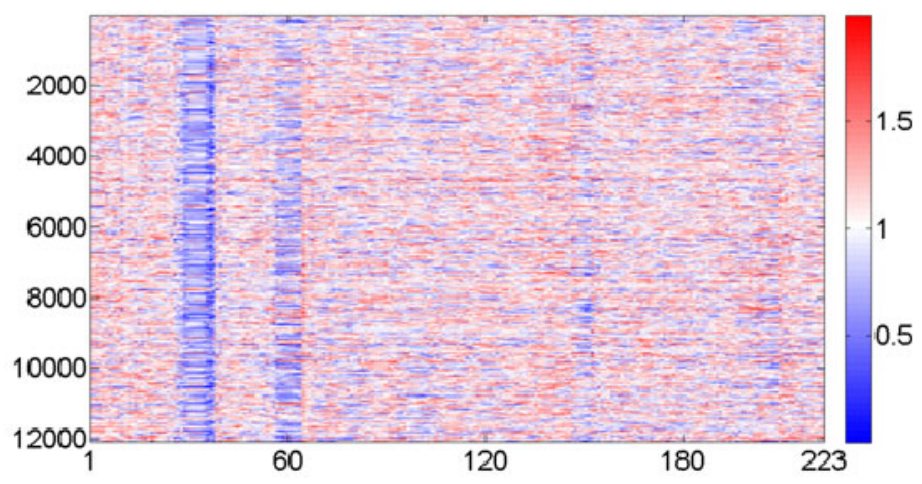

Fig. 5. Visualization of causality magnitude of all fibers in 223 sliding windows. Each row vector is the CM dynamics of one fiber through the whole time period, and each column vector is the causality magnitude state vector in that sliding window.

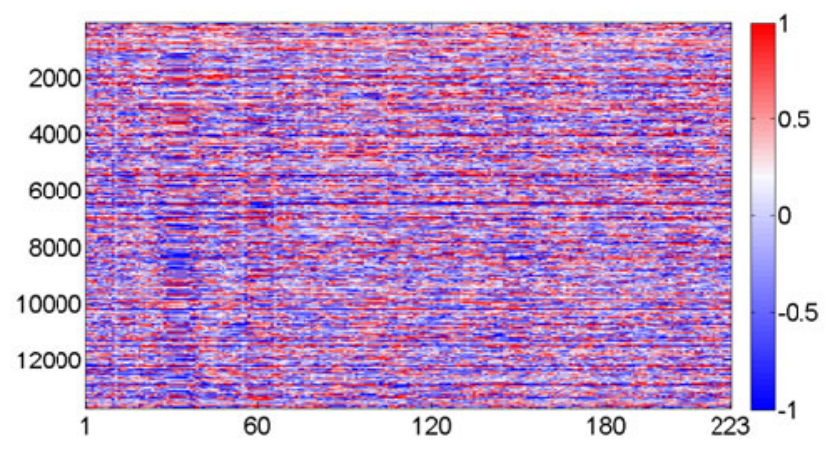

Fig. 6. Visualization of causality phase of all fibers in 223 sliding windows. Each row vector is the causality phase of one fiber through the whole time period, and each column vector is the causality phase state vector in that sliding window. 
In addition to the causality magnitude dynamics in Fig. 5, the dynamics of causality phases were also analyzed. As shown in Fig. 6, the causality phases are also changing dramatically along the scan period. In average, one fiber undergoes 50 times of phase change through the whole scan, i.e., during $22.4 \%$ of the time a fiber is changing its phase of causality. The frequency can be as high as 93 times, meaning that between every 2 time points the fiber changes its causality phase. The histogram of the frequencies of causality phase changes is shown in Fig. 7. This result reveals the dynamics in natural stimulus fMRI data and is replicated in other 4 subjects studied, imposing challenges to the assumption of temporal stationarity in traditional GCA.

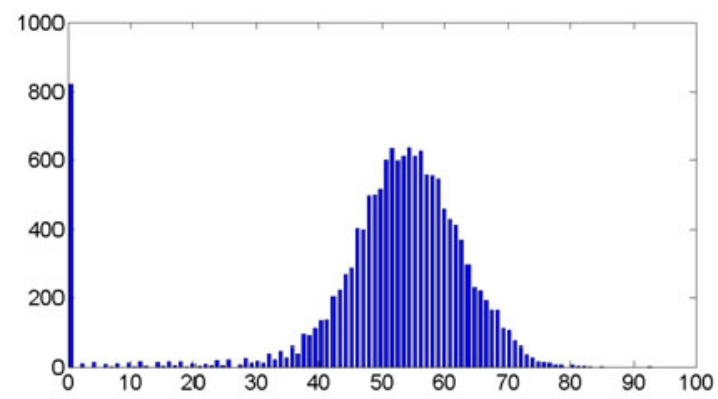

Fig. 7. Histogram of the phase change frequencies of all fibers. The phase change frequency is defined as total number of times a fiber changes its sign of causality phase.

\section{Conclusion}

This paper presented novel fiber-centered GCA studies on resting state and natural stimulus fMRI datasets for the purpose of elucidating the structural underpinnings and functional dynamics. In this approach, we extracted fMRI BOLD signals from voxel pairs connected by white matter fibers derived from DTI data, and examined their Granger causalities. Results in resting state fMRI data showed that Granger causalities on white matter fibers are significantly stronger than causalities between brain regions not fiber-connected, suggesting the structural connectivity underpinning of functional causality. In addition, the proposed approach was applied on natural stimulus fMRI data and our results suggest that Granger causalities revealed dramatic temporal dynamics, in terms of both causality magnitude and phase, warranting reexamination of the assumption of temporal stationarity of traditional GCA.

\section{References}

1. Granger, C.W.J.: Investigating Causal Relations by Econometric Models and Crossspectral Methods. Econometrica 37(3), 424-438 (1969)

2. Goebel, R., Roebroeck, A., Kim, D.-S., Formisano, E.: Investigating directed cortical interactions in time-resolved fMRI data using vector autoregressive modeling and Granger causality mapping. Magnetic Resonance Imaging 21(10), 1251-1261 (2003) 
3. Roebroeck, A., Formisano, E., Goebel, R.: Mapping directed influence over the brain using Granger causality and fMRI. NeuroImage 25(1), 230-242 (2005)

4. Ramsey, J.D., Hanson, S.J., Hanson, C., Halchenko, Y.O., Poldrack, R.A., Glymour, C.: Six problems for causal inference from fMRI. NeuroImage 49(2), 1545-1558 (2010)

5. Seth, A.K.: Causal connectivity of evolved neural networks during behavior. Network: Computation in Neural Systems 16(1), 35-54 (2005)

6. Keil, A., Sabatinelli, D., Ding, M., Lang, P.J., Ihssen, N., Heim, S.: Re-entrant projections modulate visual cortex in affective perception: Evidence from Granger causality analysis. Human Brain Mapping 30(2), 532-540 (2007)

7. Li, K., Guo, L., Li, G., Nie, J., Faraco, C., Zhao, Q., Miller, L.S., Liu, T.: Cortical surface based identification of brain networks using high spatial resolution resting state FMRI data. In: Proceedings of the 2010 IEEE International Conference on Biomedical Imaging, pp. 656-659. IEEE Press, Piscataway (2010)

8. Liu, T., Li, H., Wong, K., Tarokh, A., Guo, L., Wong, S.T.C.: Brain tissue segmentation based on DTI data. NeuroImage 38(1), 114-123 (2007)

9. Friston, K.: Causal Modelling and Brain Connectivity in Functional Magnetic Resonance Imaging. PLoS Biol. 7(2), e1000033 (2009)

10. Chang, C., Glover, G.H.: Time-frequency dynamics of resting-state brain connectivity measured with fMRI. NeuroImage 50(1), 81-98 (2010)

11. TRECVID 2005 Evaluation (2005), http://www-nlpir.nist.gov/projects/tv2005/tv2005.html

12. Richiardi, J., Eryilmaz, H., Schwartz, S., Vuilleumier, P., Van De Ville, D.: Decoding brain states from fMRI connectivity graphs. NeuroImage 56(2), 616-626 (2011)

13. Lv, J., Guo, L., Hu, X., Zhang, T., Li, K., Zhang, D., Yang, J., Liu, T.: Fiber-Centered Analysis of Brain Connectivities Using DTI and Resting State FMRI Data. In: Jiang, T., Navab, N., Pluim, J.P.W., Viergever, M.A. (eds.) MICCAI 2010. LNCS, vol. 6362, pp. 143-150. Springer, Heidelberg (2010) 